\section{Diabetes Gestacional: Persistem as Controvérsias}

$\mathbf{O}$ DIABETES Gestacional (DG) é definido como uma intolerância aos carboidratos de gravidade variável primariamente diagnosticada durante a gravidez. Esta definição admite a possibilidade de que a intolerância aos carboidratos já estivesse presente, mas não diagnosticada, antes da gestação $(1,24)$. Até o momento atual ainda não foi realizado um estudo adequadamente padronizado com o objetivo de demonstrar qual o teste ideal para o rastreamento e diagnóstico do DG $(10,11,22,28)$.

O teste de rastreamento é realizado para identificar a população de alto risco para desenvolver o diabetes na gestação. Ele difere do teste diagnóstico que realmente identifica as gestantes portadoras de DG $(9,15)$. As dúvidas em relação ao rastreamento são antigas. No passado, apenas as gestantes com história familiar de diabetes, glicosúria, filhos com peso ao nascimento superior a $4 \mathrm{~kg}$ e parto com natimortos de causa não esclarecida, eram submetidas ao teste de diagnóstico. Observou-se, contudo, que a história isoladamente teve sensibilidade de $56 \%$ para o diagnóstico de DG (16).

Atualmente são aceitos dois métodos de rastreamento e diagnóstico do DG, ou seja, um que é adotado pela Associação Americana de Diabetes, proposto pelo "National Diabetes Data Group" (NDDG) $(1,5,16,20,24)$ e outro preconizado pela Organização Mundial dd Saúde $(2,7,18)$. O primeiro é o preferido por cerca de $75 \%$ dos obstetras americanos e o segundo, muito difundido entre os europeus.

A Associação Americana de Diabetes (ADA) e o Colégio Americano de Ginecologia e Obstetrícia apoiam o teste proposto pelo "NDDG", que se baseia nos trabalhos de O'Sullivan e Mahan (1964), preconizando o rastreamento de todas as mulheres, entre 24 e 28 semanas de gestação, utilizando para o mesmo, a glicemia de 1 hora após 50 gramas de dextrosol. Caso a glicemia seja maior ou igual a $140 \mathrm{mg} / \mathrm{dl}$, deve-se, então, realizar um teste oral de tolerância a glicose com 100 gramas de dextrosol, com os seguintes valores de referência: jejum até $105 \mathrm{mg} / \mathrm{dl}$, l hora até 190 $\mathrm{mg} / \mathrm{dl}, 2$ horas até $165 \mathrm{mg} / \mathrm{dl}$ e 3 horas até $145 \mathrm{mg} / \mathrm{dl}$. Dois ou mais valores iguais ou maiores que os descritos acima, confirmam o diagnóstico de DG, enquanto um valor alterado é diagnóstico de intolerância aos carboidratos da gravidez. Há, porém, inúmeras controvérsias em relação a estes parâmetros $(3,5,6,10-13,17,21,22,28-32)$ :

- quais gestantes devem ser rastreadas?

- quais os valores de corte do rastreamento?

- qual a época de realização do rastreamento?

- o rastreamento pode ser utilizado como diagnóstico?

- quais os valores limítrofes da curva? como realizar a curva?

Alguns estudos (1,21,25,29-31) têm demonstrado que rastrear mutheres sem fatores de risco seria desnecessário pela baixa prevalência de DG neste grupo, com custo muito elevado para o sistema de saúde. Desta perspectivas

\author{
Anelise I. Nogueira \\ Andréa S. Fontenele \\ Regina A. de Aguiar
}

Serviço de Endocrinologia ( $A I N, A S F)$, Departamento de Clínica Médica e Pré-natal de Alto-Risco (RAA), Departamento de Ginecologia e Obstetricia, Hospital das Clinicas da Universidade Federal de Minas Gerais, Belo Horizonte, $M G$. 
forma, preconizam que o teste não seja universal e, sim, baseado na presença de dois ou mais fatores de risco. São consideradas de baixo risco para desenvolver o DGl, aquelas gestantes pertencentes a um grupo étnico com baixa prevalência de DG; aquelas sem história de diabetes em parentes de primeiro grau; idade menor que 25 anos, peso normal antes da gravidez; ausência de história de DG; passado obstétrico favorável $(1,16)$.

Tanto o valor de corte do rastreamento, quanto os valores da curva são questionados por alguns autores $(3,5,6,11-13,22,25,32)$. Sugere-se adotar para o rastreamento valores de corte mais baixos: entre 130 e $135 \mathrm{mg} / \mathrm{dl} \mathrm{e}$, caso haja dois ou mais fatores de risco, o valor de $125 \mathrm{mg} / \mathrm{dl}$. Quanto aos valores da curva, as divergências também são inúmeras. Classicamente, a mais comentada é aquela de Carpenter e Coustan, que sugerem valores bem mais baixos do que os atualmente adotados pela ADA, ou seja, jejum: $95 \mathrm{mg} / \mathrm{dl}$, l hora: $180 \mathrm{mg} / \mathrm{dl}, 2$ horas: $155 \mathrm{mg} / \mathrm{dl}$ e 3 horas: $140 \mathrm{mg} / \mathrm{dl}$. No $4^{\text {th }}$ Workshop-Conference em DG, recomendou-se a adoção dos valores acima, para interpretação do TOTG, realizado após a ingestão de 100 gramas de dextrosol $(1,4,16)$.

As gestantes consideradas de alto risco para desenvolver DG devem ser rastreadas já na primeira consulta de pré-natal, conforme vem sendo preconizado por alguns autores $(16,17,28)$ que seguem as orientações da ADA, sempre com a repetição do exame entre 24 e 28 semanas de gestação no caso de negatividade do teste inicial. Há também aqueles que defendem a repetição do teste com 32 semanas de gestação, naquelas gestantes consideradas de alto risco, caso os anteriores sejam negativos (5).

Outros autores (13), também seguidores das orientações da ADA, consideram que o teste de rastreamento tem valor diagnóstico, variando apenas o nível de corte, sendo preconizado por alguns o nível de $140 \mathrm{mg} / \mathrm{dl}(12)$, enquanto outros $(3,13)$ utilizam valores entre $186 \mathrm{mg} / \mathrm{dl}$ e $216 \mathrm{mg} / \mathrm{dl}$. Estes valores dispensariam a realização da curva.

Não bastassem tantas controvérsias dentro da $\mathrm{ADA}$, resta ainda o fato de que a Organização Mundial de Saúde aplica e preconiza outro método de rastreamento e diagnóstico do DG $(2,7,14,16,18,19,23,25)$. Neste esquema, a glicemia de jejum deve ser realizada no início do pré-natal; se $>126 \mathrm{mg} / \mathrm{dl}$, em duas ocasiões, é estabelecido o diagnóstico de DG; valores entre $110 \mathrm{e} 126 \mathrm{mg} / \mathrm{dl}$, indicam a realização do teste oral de tolerância a glicose (TOTG), com $75 \mathrm{~g}$ de dextrosol. Isto significa que, se o valor da $2^{\text {a }}$ hora estiver maior ou igual a $140 \mathrm{mg} / \mathrm{dl}$, é estabelecido o diagnós- tico de DG. Se as glicemias em jejum estiverem abaixo de $110 \mathrm{mg} / \mathrm{dl}$, o TOTG deverá ser realizado entre $24 \mathrm{e}$ 28 semanas de gestação, adotando-se também os mesmos critérios.

Aqui, também, há inúmeras controvérsias:

- Alguns autores preconizam que o diagnóstico de DG seja realizado com glicemia 2 horas após $75 \mathrm{~g}$ de dextrosol, com valores maiores que $140 \mathrm{mg} / \mathrm{dl}$ $(11,14)$;

- Alguns preconizam outros valores de corte para glicemia de jejum: se o nível em jejum for maior ou igual a $90 \mathrm{mg} / \mathrm{dl}$ em duas ocasiões, é firmado o diagnóstico de DG (18), outros níveis para glicemia de jejum também são sugeridos: 105,110 e até mesmo $140 \mathrm{mg} / \mathrm{dl}(3, \mathrm{ll})$;

- Consenso Brasileiro de Diabetes Gestacional preconiza um rastreamento precoce, utilizando a glicemia de jejum $(23,26)$ já na primeira consulta de pré-natal: glicemia de jejum maior ou igual a 85 $\mathrm{mg} / \mathrm{dl}$ serve como rastreamento pois, nesta situação, indica-se o TOTG, de 2 horas, realizado com $75 \mathrm{~g}$ de dextrosol. Os parâmetros utilizados para o teste são aqueles adotados pela WHO, ou seja uma glicemia da segunda hora maior ou igual a $140 \mathrm{mg} / \mathrm{dl}$ é diagnóstico de DG. Nestas pacientes indica-se também a realização de glicemia de jejum novamente com 20 semanas, seguindo-se as recomendações acima. No caso de glicemia de jejum $<85 \mathrm{mg} / \mathrm{dl}$, o teste deve ser realizado entre 24 e 28 semanas de gestação e repetido com 32 semanas em pacientes com mais fatores de risco para DG.

Apesar de tantas controvérsias, sabe-se que o DG é uma entidade clínica que coloca em risco o feto e o recém-nascido. Sabe-se, também, que a médio c longo prazo poderá acarretar em obesidade e risco de diabetes tipo 2 para estas crianças $(27,28)$. Além disto, é preditor de diabetes tipo 2 para estas mulheres $(8,28)$. Portanto, é fundamental a busca do exame de realização racional para o rastreamento e diagnóstico do $\operatorname{DG}(1,16)$.

Como se afirmou anteriormente, do ponto de vista conceitual, um teste de rastreamento pretende identificar junto à população geral um grupo de pessoas de risco elevado para o desenvolvimento de uma determinada doença. Todo teste de rastreamento alterado deve ser seguido de um teste diagnóstico. As características básicas de um bom teste de rastreamento são basicamente: simplicidade, custo reduzido, boa tolerância, boa sensibilidade e reprodutibilidade. 
Ao escolher um teste para ser utilizado na prática clínica, o profissional deve estar sempre atento às características específicas de cada um deles, tendo em mente as suas vantagens e limitações. Todo teste "novo" deve ser introduzido na clínica diária após ter seus resultados validados por protocolos de pesquisa eticamente conduzidos. Além disso, é importante que o profissional procure estar sempre atento às novas orientações e modificações no processo de rastreamento e diagnóstico do $\mathrm{DG}$, buscando o objetivo comum que é garantir um bom prognóstico materno e perinatal $(16,28)$.

\section{AGRADECIMENTOS}

Agradecemos ao Prof. Leonardo Maurício Diniz, pelo apoio na execução deste trabalho.

\section{REFERÊNCIAS}

1. American Diabetes Association: Gestational diabetes mellitus (Position Statement) Diabetes Care 1998;supp 1: S60-61.

2. Alberti KGMM, Zimemet $\mathrm{PZ}$ for the WHO consultation. Definition, diagnosis and classification of diabetes and its complications. Part 1 : diagnosis and classificativii ut diabetes mellitus. Provisional report of a WHO consultation. Diabetic Medicine 1998;15:539-53.

3. Bobrowski RA, Bottoms SF, Micallef JA, Dombrowski MP. Is the 50-gram glucose screening test ever diagnostic? J Matern Fetal Med 1996;5:317-20.

4. Coustan DR, Carpenter MW. The diagnosis of gestational diabetes. Diabetes Care 1998;supp 2:21.

5. Coustan DR. Screening and diagnosis of gestational diabetes. Semin Perinatol 1994;18:407-13.

6. Coustan DR. Commentary on the O'Sullivan and Mahan criteria. Diabetes Spectrum 1992;5:31-3.

7. Deerochanawong $C$, Putiyanum $C$, Wongsuryrat $M$, Serirat S, Jinayon P. Comparison of National Diabetes Data Group and World Health Organization criteria for detecting gestational diabetes mellitus. Diabetologia $1996 ; 39 ; 1070-3$

8. Feig DS, Chen E, Naylor D. Self perceived health status of women three to five years after the diagnosis of gestational diabetes: A survey of cases and matched controls. Am J Obstet Gynecol 1998; 178: 386-93.

9. Gordis L. Epidemiology. Chicago:WB Saunders Co. 1996

10 Greene MF. Screening for gestational diabetes (Editorial). N Eng J Med 1997:337: 1625-6.

11 Humphrey MD. Gestational diabetes mellitus: screening controversy. MJA 1998;168:138-9.

12. Jovanovic Peterson L, Bevier W. Peterson CM. The Santa Barbara County Health Care Services program: birth weight change concomitant with screening for and treatment of glucose-intolerance of pregnancy: a potential cost-effective intervention? Am J Perinatol $1997: 14: 221-8$.

13. Landy HJ, Gomez-Marin O, O'Sullivan MJ. Diagnosing gestational diabetes mellitus: use of a glucose screen without administering the glucose tolerance test. Obstet Ginecol 1996:87:395-400.

14. Lao $\Pi$, Lee CP. Gestational "impaired glucose tolerance": should the cut-of be raised to $9 \mathrm{mmol} 1(-1)$ ? Diabet Med 1998;15:25-9.

15. Lilienfeld DE, Stolley PD. Foundations of Epidemiology. 3id ed. Oxford University Press, 1994.

16. Metzger BE, Coustan DR . Committee Organizaning: Summary and recomendations of the Fourth International Workshop-Conference on gestational Diabetes Mellitus. Diabetes Care 1998;21(suppl 2):B161-B167.

17. Meyer WJ, Carbone J, Gauthier DW, Gottmann DA. Early gestational glucose screening and gestational diabetes. J Reprod Med 1996;41:675-9.

18. Moses RG. Russel KG. Scheir GM, Moses M. The $75 \mathrm{~g}$ glucose tolerance test in pregnancy. Diabetes Care 1998:21:1807-11.

19. Nasrat HA, Sabbagh SA, Ardawi MSM. New criteria for interpretation of the $75 \mathrm{~g}$ oral glucose tolerance test in pregnancy. Metabolism 1990;39:51-7.

20. National Diabetes Data Group: Classification and diagnosis of diabetes mellitus and other categories of glucose intolerance. Diabetes 1979;28: 1039-57

21. Naylor CD, Sermer M, Chen E, Farine AD. Selective screening for gestational diabetes mellitus. $N$ Eng J Med 1997:337:1591-6.

22. Pettit JD. Gestational diabetes mellitus. Who to test. How to test. Diabetes Care 1998;21:1789

23. Reichelt AJ, Spichler ER, Branchtein L, Nucci LB, Franco LJ, Schmidt Ml. Fasting plama glucose is a useful test for the detection of gestational diabetes. Diabetes Care $1998 ; 21: 1246-9$

24. Report of Expert Committee on the diagnoses and classification of diabetes mellitus. Diabetes care 1997;20: $1183-97$.

25. Samad N. Hassan JA, Shera AS. Gestational diabetes mellitus: screening in a developing country. J Pak Med Assoc 1996:46:249-52

26. Schmidt MI, Reichelt AJ. Is fasting glucose a useful screening test for gestational diabetes mellitus and ges tational impaired glucose tolerance? $56^{\text {th }}$ Annual Meeting and Scientific Sessions of the Diabetes Association. Diabetes 1996:45(suppl 2): 176 A (abstract 645).

27. Silverman BL, Metzger BE, Cho NH, Loeb CA. Impaired glucose tolerance in adolescent offspring of diabetic mothers. Diabetes Care 1995;18:611-7.

28. Soares JAC, Dornhorst A, Beard RW. The case for screening for gestational diabetes. BMJ 1997;315: 737-9.

29. Solomon CG. Willet WC, Rich-Edwards J, et al. Variability in diagnostic evaluation and criteria for gestational diabetes. Diabetes Care 1996;19:12-16

30. Stephen RC. Screening for gestational diabetes mellitus. Diabetes Care 1998:21:supp 2 
31. Weeks JW. Major CA, Veciana M, Morgan MA. Gestational diabetes: Does the presence of risk factors influence perinatal outcome? Am J Obstet Gynecol $1994,171: 4,1003-7$.

32. Yalçin HR. Threshold value of glucose screening tests in pregnancy. Am J Perinatol 1996;13:317-20.

\section{Endereço para correspondência:}

Anelise I. Nogueira

Av. Alfredo Balena, $189-15^{\circ}$ andar, sala 1501

30130-100 Belo Horizonte, MG

Fax: (031) 274-4331

E-mail: anelise@pobox.com 